Supporting Information for

\title{
Self-powered, one-stop and multifunctional implantable triboelectric active sensor for real-time biomedical monitoring
}

Ye $\mathrm{Ma}^{\dagger,}{ }^{\perp}$, Qiang Zheng ${ }^{+,}{ }^{\perp}$, Yang Liu ${ }^{\dagger,}{ }^{\perp}$, Bojin Shit, Xiang Xue ${ }^{\dagger}$, Weiping Jit, Zhuo

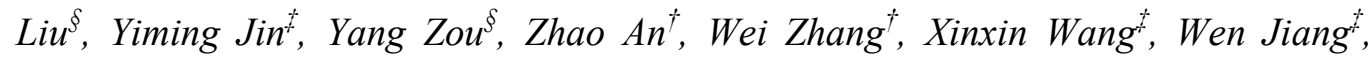

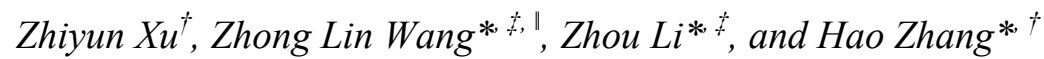

$\dagger$ Institute of Cardiothoracic Surgery at Changhai hospital, Second Military Medical University, Shanghai 200433, PR China

¥Beijing Institute of Nanoenergy and Nanosystems, Chinese Academy of Science, Beijing 100083, PR China

$\S$ School of Biological Science and Medical Engineering, Beihang University, Beijing 100191, PR China

\|School of Materials Science and Engineering, Georgia Institute of Technology, Atlanta, GA 30332, USA

\section{Corresponding Author}

* (Z.L.W.) E-mail: zhong.wang@mse.gatech.edu

*(Z.L.) E-mail: zli@,binn.cas.cn

* (H.Z.) E-mail: zhanghao@smmu.edu.cn 


\section{Experimental methods}

Device structure and fabrication: The as-fabricated iTEAS was composed of triboelectric layers, electrodes, spacers and encapsulation layers. In detail, a thin-film nanostructured PTFE (n-PTFE, $50 \mu \mathrm{m}$ ) was employed as a triboelectric layer to increase the output signals. A Kapton (polyimide) film $(150 \mu \mathrm{m})$ was fixed on n-PTFE layer serving as a substrate for its excellent elasticity and strength so that triboelectric layer can easily contact and separate. An ultrathin Au layer (50 nm) was deposited on the back side of the Kapton film to form one of the electrodes. Al film $(100 \mu \mathrm{m})$ was used as both another triboelectric layer and electrode. In addition, it is worth noting that the spacers combined with an elastic titanium strip were integrated on Kapton film to guarantee the contact and separation more effectively. The aforementioned structure was then encapsulated in several packages. PTFE was used as the first encapsulation material which has been widely used in medical devices. A flexible PDMS layer was then deployed by spin-coating to enhance the leakproofness. In order to further increase the stability of the device and avoid potential erosion in physiological environment, a Parylene film was deposited to form a high density and hole-free coating layer.

In Vivo Study: The animal was handled in accordance with the "Shanghai Administration Rule of Laboratory Animal" and the Institutional Animal Care and Use Committee (IACUC) approved protocol of the Animal Care Center at the Second Military Medical University. Male Yorkshire porcine $(30 \mathrm{~kg}$ ) fasted for 12 hours prior to surgery. Briefly, the animal was anesthetized with an injection of ketamine 
$(8 \mathrm{mg} / \mathrm{kg}, \mathrm{IM})$ followed by propofol $(1 \mathrm{mg} / \mathrm{kg}, \mathrm{IV})$, then intratracheally intubated and ventilated in a rate of 12 cycles per minute ( 5 seconds per cycle). Anesthesia was maintained with $1.0 \%$ isoflurane. An arterial pressure catheter was placed in the right femoral artery and connected to a multi-channel data acquisition (DAQ) system (PowerLab 4/35 AD Instruments, AUS) through the transducer mentioned above. The surface ECG was also connected to the DAQ system. Then the device was implanted and fixed to the pericardium facing the ventricular wall with a 4-0 prolene suture. The electrodes of the device were connected to the DAQ system though lead wires maintained protruding the chest wall for measuring the electric output. A $100 \mathrm{M} \Omega$ probe was used to directly measure the voltage while a $1 \mathrm{M} \Omega$ probe for the current. LabChart 8 software (AD Instruments, Sydney, NSW, Australia) was used to record the acquired data.

Animal models: Epinephrine $(0.1 \mathrm{mg} / \mathrm{ml}$, IV) was administrated as $\beta$-receptor agonist to increase the heart rate to simulate the status of tachycardia. Two pacing leads were sutured into the epicardium and connected to an electronic cardiac pacemaker (Saint Jude Medical, US) which was set to 50 beat per minute (bpm) in enforce mode for mimicking the frequent ventricular premature contractions. To evaluate the monitoring function for respiration, the device was implanted to the left lateral, right lateral and posterior wall of heart.

Histological evaluation of in vivo biocompatibility: After 2 weeks of implantation, the animals were euthanized and myocardium tissues were excised from implantation site and distal non-implantation site as a control. Followed by $4 \%$ neutral-buffered 
formalin fixation and paraffin embedding, tissue sections $(5 \mu \mathrm{m})$ were prepared for histologic analysis including hematoxylin and eosin (H\&E), Masson's trichrome and immunohistochemical stains. H\&E stain was applied to identify the infiltration of inflammatory cells and damage of cardiac muscles. Masson's trichrome, which can clearly distinguish cardiomyocytes (pink red) and assess the level of hyperplastic collagen fiber (blue), was conducted based on a commercial Masson's trichrome kit purchased from Leagene Biotechnology (Beijing, China). For immunohistochemistry, tissue sections were incubated with anti-cleaved-caspase 3 (1:200 dilution, Cell Signaling Tech., MA) under $4 \square$ overnight after heat-mediated antigen retrieval and blocking by $5 \%$ BSA. Followed by incubation in biotinylated secondary antibody for 15 minutes, the sections were detected using $\mathrm{DAB}$ as chromogen and then counterstained with hematoxylin.

\section{In vitro self-powered ability of the iTEAS}
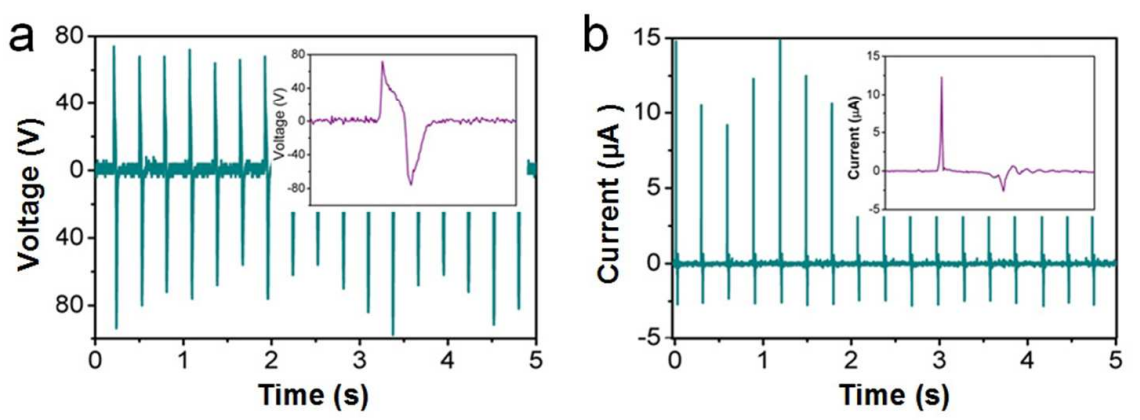

Figure S1. (a) Typical open-circuit voltage and (b) short-circuit current of the iTEAS were generated using an external linear motor. The insets show their respective enlarged waveforms. 


\section{Implantation of the iTEAS}
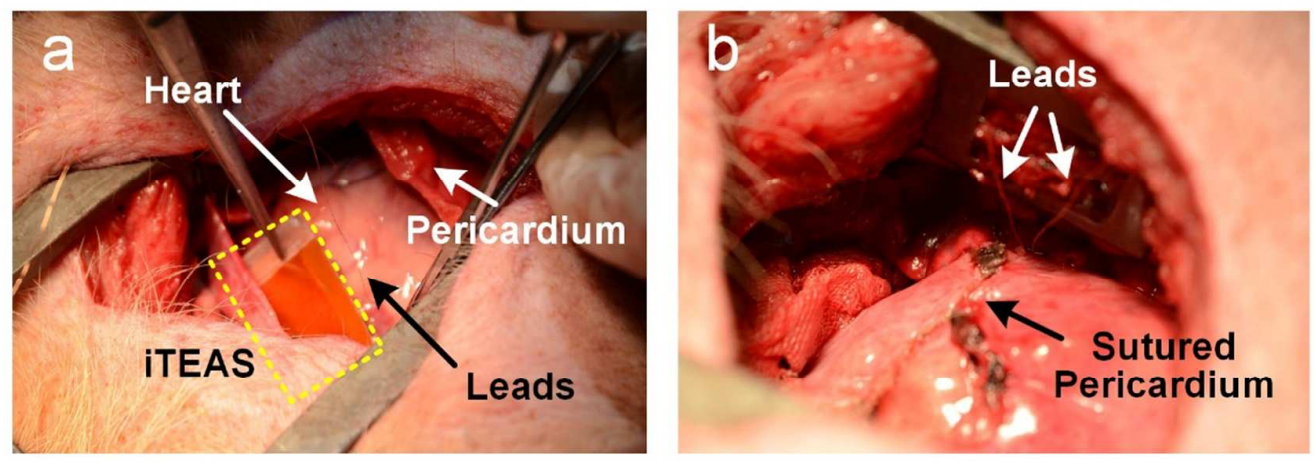

Figure S2. (a) The device (dashed box highlighted) was inserted into the space between pericardium and epicardium. Then the iTEAS was fixed onto the pericardium. (b) The pericardium was closed with running suture.

\section{Further inspection of output voltage and blood pressure} waveforms.

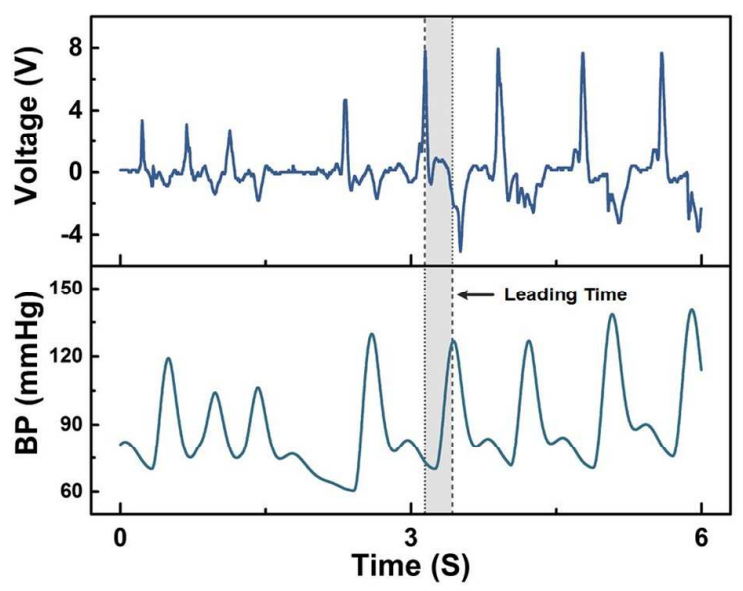

Figure S3. Further inspection demonstrated the synchronicity between waveforms of output voltage and blood pressure. Highlighted region represents time interval between the peak of output voltage and the correspondent peak of BP waveform. 


\section{Table S1: Monitoring of respiratory phases and I/E ratio}

\begin{tabular}{cccc}
\hline $\mathbf{R C}$ & $\mathbf{T}_{\mathbf{I}}(\mathbf{s})$ & $\mathbf{T}_{\mathbf{E}}(\mathbf{s})$ & $\mathbf{I} / \mathbf{E}$ ratio \\
\hline$\# 1$ & 3.095 & 2.035 & 1.521 \\
$\# 2$ & 2.993 & 2.017 & 1.484 \\
$\# 3$ & 3.106 & 2.043 & 1.520 \\
$\# 4$ & 2.892 & 2.025 & 1.428 \\
$\# 5$ & 3.053 & 2.070 & 1.475 \\
$\# 6$ & 3.072 & 2.067 & 1.486 \\
\hline
\end{tabular}

Data were obtained from randomly selected respiratory cycles when the iTEAS fixed upon the left lateral wall of the heart. RC, respiratory cycle; $\mathrm{T}_{\mathrm{I}}$, measured durations of inspiration phase; $\mathrm{T}_{\mathrm{E}}$, measured durations of expiration phase; $\mathrm{I} / \mathrm{E}$ ratio, the ratio of duration of inhalation phase to exhalation phase.

\section{Discussion on the source and management of small waves of voltage outputs}

Throughout our work, there constantly exists relatively small peaks between two prominent peaks at each cycle in most of the voltage data. According to the working principle of the implanted triboelectric sensor and our data, we attribute the occurrence of these relatively tiny waves to the following reasons: 1) Different parts of heart are not totally synchronously contracted for that the heart is not a simple and perfect balloon-like shape and the wall of ventricle are composed of three layers of cardiac muscle fibers which is arranged in three different directions; 2) Rapid blood flows filling the heart form turbulence when passing cardiac valves and hitting inner 
wall of the heart, which will lead to subtle oscillation of the heart; 3) The surface of the heart is unsmooth and non-planar, which composes of myocardium, pulsatile coronary artery accompanied by veins and irregular-shaped fat. Therefore, the behaviors of the relatively tiny waves vary throughout our work and can be easily influenced by the position of the sensor to the heart, individual variances among different experimental animals and different states of the heart during our experiments. These small waves even occasionally disappeared or could be hardly observable (e.g. Fig. 2 b and $3 d$ ). In most cases, the small waves behave in a fixed pattern in a short period, which is similar to their neighboring prominent waves. For example, periodical fluctuations of the small peaks were identified when placing the device on the left lateral wall of the heart (Fig. 4c), indicating that these small peaks can potentially be utilized for respiratory monitoring. However, due to their unstable and vulnerable performances, we chose to ignore the small waves or peaks as "background noise signals" and focus on the prominent waves in the present works. In practical application, a threshold can be set up to eliminate the interference brought by the small waves when analyzing the output signals of the proposed active sensor. 